\title{
3D printer-based novel intensity-modulated vaginal brachytherapy applicator: feasibility study
}

\author{
Fatih Biltekin, PhD, Husnu Fadil Akyol, MD, Melis Gültekin, MD, Ferah Yildiz, MD \\ Department of Radiation Oncology, Faculty of Medicine, Hacettepe University. Ankara, Turkey
}

\begin{abstract}
Purpose: To design a novel high-dose-rate intracavitary applicator which may lead to enhanced dose modulation in the brachytherapy of gynecological cancers.

Material and methods: A novel brachytherapy applicator, auxiliary equipment and quality control phantom were modeled in SketchUp Pro 2017 modeling software and printed out from a MakerBot Replicator Z18 three-dimensional printer. As a printing material polylactic acid (PLA) filament was used and compensator materials including aluminum, stainless-steel and Cerrobend alloy were selected according to their radiation attenuation properties. To evaluate the feasibility of the novel applicator, two sets of measurements were performed in a Varian GammaMed iX Plus highdose rate iridium-192 (192 Ir) brachytherapy unit and all of the treatment plans were calculated in Varian BrachyVision treatment planning system v.8.9 with TG43-based formalism. In the first step, catheter and source-dwell positioning accuracy, reproducibility of catheter and source positions, linearity of relative dose with changing dwell times and compensator materials were tested to evaluate the mechanical stability of the designed applicator. In the second step, to validate the dosimetric accuracy of the novel applicator measured point dose and two-dimensional dose distributions in homogeneous medium were compared with calculated data in the treatment planning system using PTW VeriSoft v.5.1 software.

Results: In mechanical quality control tests source-dwell positioning accuracy and linearity of the designed applicator were measured as $\leq 0.5 \mathrm{~mm}$ and $\leq 1.5 \%$, respectively. Reproducibility of the treatment planning was $\geq 97.7 \%$ for gamma evaluation criteria of $1 \mathrm{~mm}$ distance to agreement and 1\% dose difference of local dose. In dosimetric quality control tests, maximum difference between measured and calculated point dose was found as 3.8\% in homogeneous medium. In two-dimensional analysis, the number of passing points was greater than $90 \%$ for all measurements using gamma evaluation criteria of $3 \mathrm{~mm}$ distance to agreement and $3 \%$ dose difference of local dose. Conclusions: The novel brachytherapy applicator met the necessary requirements in quality control tests.

Key words: intensity-modulated brachytherapy, novel applicator, gynecological cancers.

\section{Purpose}

Intracavitary brachytherapy (BRT) has a significant role in the treatment of gynecologic malignancies $[1,2,3,4,5,6,7]$. It offers the major advantage of rapid dose fall off that provides better dose sparing of the surrounding tissues and critical organs adjacent to the target $[8,9]$. The recent $\mathrm{Na}-$ tional Cancer Database analysis showed that consolidation BRT was a critical component for locally advanced cervical cancer [5]. Additionally, vaginal BRT, alone or in combination with external beam radiotherapy (EBRT), is used in a selected group of patients with cervical, vaginal or vulvar cancer [10]. Recently there is growing interest in the use of vaginal BRT alone as adjuvant treatment after surgery in endometrial carcinoma (EC) [3].

The vaginal cuff is the main location of local relapses after surgery in EC and it was shown that adjuvant vagi- nal BRT alone produced similar local control and survival rates compared to external pelvic radiotherapy in patients with intermediate or high-intermediate risk factors [11]. The most commonly used applicator for intracavitary BRT in EC is a centrally located single channel (CSC) vaginal cylinder and the dose is generally prescribed to either the surface of the cylinder or $0.5 \mathrm{~cm}$ in depth. These applicators can also be used for primary vaginal cancer or recurrent vaginal tumors either alone or combined with EBRT. However, these CSC applicators offer limited possibilities in the planning optimization due to their radial symmetry of dose distribution and can be used only in tumor volumes having thickness or depths less than $5 \mathrm{~mm}$ from the applicator surface. To improve the quality of treatment in deeper and asymmetric tumors, several multichannel (MC) applicators have been developed. In these commercial MC applicators, additional peripheral channels added
Address for correspondence: Fatih Biltekin, PhD, Department of Radiation Oncology, Hacettepe University Faculty of Medicine, 06100, Ankara, Turkey, phone: +903123052900,

e-mail: fatih.biltekin@hacettepe.edu.tr
Received: 28.10.2019

Accepted: 18.12 .2019

Published: 28.02.2020 
to the central axis provide more conformal dose distributions up to $1 \mathrm{~cm}$ depth. Even with these MC applicators, vaginal lesions thicker or deeper than $1 \mathrm{~cm}$ cannot be covered effectively without an additional unwanted dose to the surrounding normal tissues such as bladder, rectum or sigmoid colon. Therefore standard MC applicators lose their advantage in these cases and interstitial BRT is frequently required $[12,13,14,15]$, which needs expertise in the field, general anesthesia and hospitalization.

In the present study we aimed to design a novel highdose-rate (HDR) intensity-modulated brachytherapy (IMBRT) applicator as an alternative to interstitial BRT in vaginal tumors thicker than $1 \mathrm{~cm}$ and in cervical tumors with gross residual disease after EBRT or with difficult anatomy in order to cover the tumor adequately while decreasing the dose to the surrounding normal tissues. In addition to the novel design, this study investigates the adaptation of current three-dimensional (3D) printer technology to medical applications.

When the novel applicator meets all requirements for clinical use (e.g. safety, sterilization, health and environmental protection), the workflow for the use of a novel applicator in the treatment can be divided into five steps: 1) making a preliminary patient-specific computed tomography (CT) simulation and preplan to determine the type, length and position of the compensator materials using an automated contouring template, 2) scanning treatment planning $\mathrm{CT}$ with defined preplan conditions including type, length and position of the materials using an extended CT scale and metal artifact reduction algorithm, 3) transferring planned dwell time and position of the radioactive source from preplan $\mathrm{CT}$ to new planning $\mathrm{CT}, 4)$ controlling transferred treatment plan parameters and performing necessary arrangements to improve the quality of treatment planning, 5) approval of the treatment plan and transfer to the treatment machine.

\section{Material and methods}

This novel IMBRT applicator was designed by a senior radiation oncologist (H.F.A.) and a medical physicist (F.B.). All of the treatment planning simulations and quality control (QC) tests were performed using Varian BrachyVision v.8.9 (Varian Medical Systems, Palo Alto, CA) treatment planning system (TPS) with TG-43-based

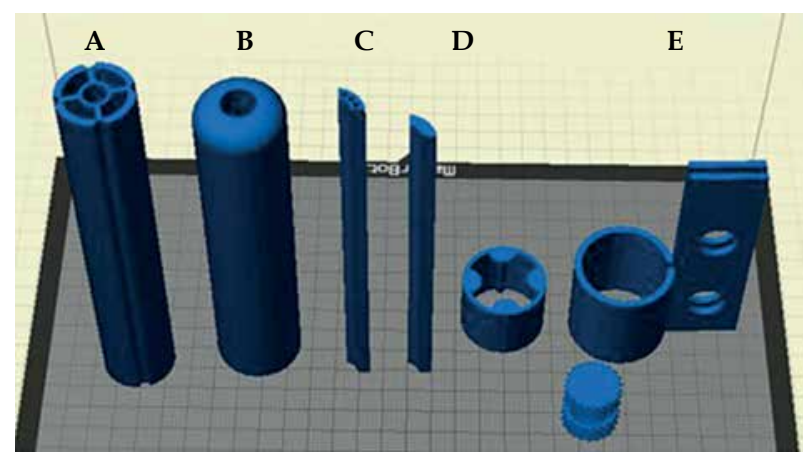

Fig. 1. Novel IMBRT applicator modeling A) internal body, B) external body, C) source transfer channels, D) compensator materials and E) fixation tools formalism and a Varian GammaMed iX Plus HDR ${ }^{192}$ Ir BRT unit (Varian Medical Systems, Palo Alto, CA), respectively. To evaluate the feasibility of this novel IMBRT vaginal applicator, investigations were carried out following 4 main steps: 1) material selection for applicator, phantom, auxiliary fixation tools and compensators, 2) modeling and printout of the products, 3) evaluating printing accuracy of the 3D printer, 4) performing mechanical and dosimetric QC tests.

\section{Material selection}

In the production of the applicator, auxiliary fixation tools and QC phantom polylactic acid (PLA) filament with a density of $1.25 \mathrm{~g} / \mathrm{cm}^{3}$ was used as the printing material. PLA is suitable for medical applications since it is biocompatible and it can be sterilized with ethylene oxide (EtO) and gas plasma. Aluminum (density: $2.5 \mathrm{~g} / \mathrm{cm}^{3}$ ), stainless steel (density: $8.0 \mathrm{~g} / \mathrm{cm}^{3}$ ) and Cerrobend alloy (density: $9.4 \mathrm{~g} / \mathrm{cm}^{3}$ ) were selected as compensator materials according to their radiation attenuation characteristics. The main purpose of the directional compensator is not to provide complete shielding of critical organs such as the rectum and bladder near the target volumes but to modulate dose distribution to reduce the critical organ dose and increase target coverage.

\section{Modeling and 3D printing}

All of the products were modeled in SketchUp Pro 2017 (Trimble Inc., Sunnyvale, CA) 3D modeling program. Additionally, Meshmixer software (Autodesk, San Rafael, USA) was used to make the necessary arrangements and to save the file in .stl format for 3D printing.

The IMBRT applicator was modeled in two different diameters of 30 and $35 \mathrm{~mm}$. The total length of the applicator was $190 \mathrm{~mm}$. The designed applicator had five removable parts, i.e. the external body, internal body, source transfer channels, compensator materials and auxiliary fixation tools, as illustrated in Figures 1 and 2. The applicator had four chamber cavities allowing placement of source transfer channels or compensator materials according to the target and critical organs location and one central channel for tandem insertion in the internal body. In the peripheral channels the source was encapsulated within a 5-French plastic catheter. The special design of

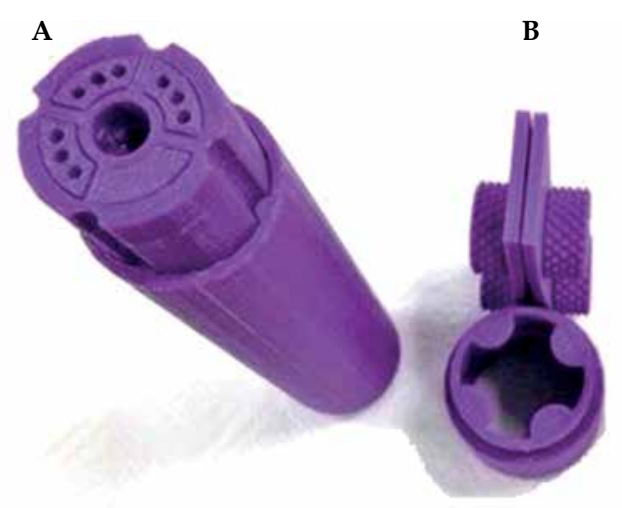

Fig. 2. Prototype of novel IMBRT applicator A) applicator with a diameter of $35 \mathrm{~mm}$ and $\mathbf{B}$ ) auxiliary fixation tools 
the applicator allows different combinations of the source transfer channels and desired lengths of compensator materials during application and treatment. Fixation tools (Figures 1E and 2B) compatible with the BRT treatment couch were also designed to fix the removable part of the applicator. Additionally, the specific QC phantom and applicator were modeled (Figure 3 ) and printed out to perform all QC tests in the same measurement setup. The output of the products except for compensator materials was taken using PLA filament in a MakerBot Replicator Z183D printer (MakerBot Industries, Brooklyn, USA) and modeled applicators were printed by choosing $0.2 \mathrm{~mm}$ print resolution, 2 layer support material and 100\% infill percentage.

\section{Printing accuracy of $3 D$ printer}

Calibration of the 3D printer was performed before each printing process to improve the quality of the printout. Printing accuracy of the product was also evaluated through physical measurements using a Vernier caliper with a resolution of $0.1 \mathrm{~mm}$. Physical measurements were performed at multiple positions along the prints and all measurements were compared with the designed dimensions of the product in modeling software. Additionally, total printing time was evaluated for all parts of the modeled applicator.

\section{Mechanical QC tests}

Mechanical QC tests were performed in three steps: 1) catheter and source dwell positioning accuracy, 2) reproducibility of catheter and source position for different step sizes, and 3) linearity of relative dose with changing dwell time and compensator materials. In the first step, 5-French catheters were inserted into the peripheral

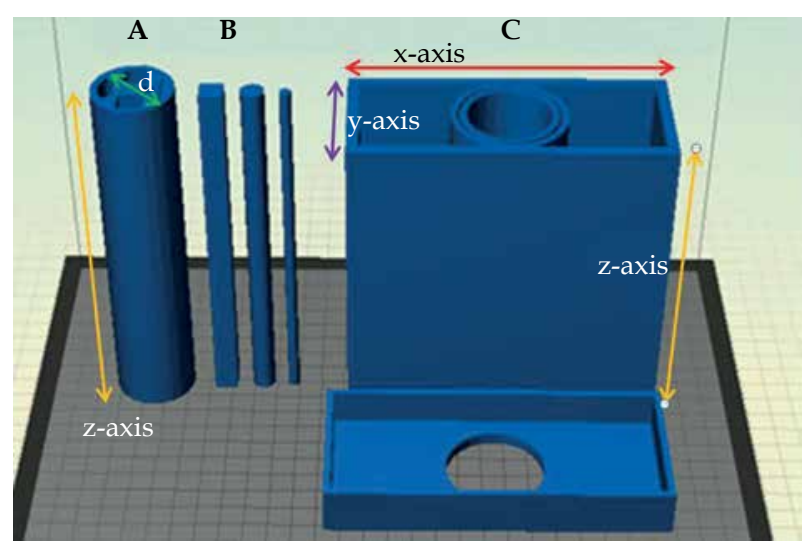

Fig. 3. 3D printed A) $Q C$ applicator $(\mathrm{d}=35 \mathrm{~mm}$ and $\mathrm{z}=190$ $\mathrm{mm}$ ) and $\mathbf{B}$ ) compansator material with different shape and C) QC phantom ( $x=150 \mathrm{~mm}, \mathrm{y}=51 \mathrm{~mm}$ and $\mathrm{z}=165 \mathrm{~mm}$ )

transfer channel of the designed applicator and a source dwell positioning accuracy test was performed with Kodak EDR2 radiographic film (Eastman Kodak Company, Rochester, NY, USA). To verify the dwell positioning accuracy of the radioactive source, $\mathrm{x}$-ray markers were placed into the catheter and reference radiography was acquired to image the reference $x$-ray marker position. Next, an autoradiograph was performed with radioactive source and irradiated dwell positions were compared with the reference dummy marker position. The tolerance value for source dwell positioning accuracy was less than $2 \mathrm{~mm}$. Additionally, the actual catheter insertion position obtained with autoradiography was compared with the reconstructed catheter position in the TPS. In the second step, reproducibility of the catheter and source position for three different step sizes of $1,0.5$ and $0.1 \mathrm{~cm}$ was tested using the 2D-Array seven29 (PTW-Freiburg, Ger-

A

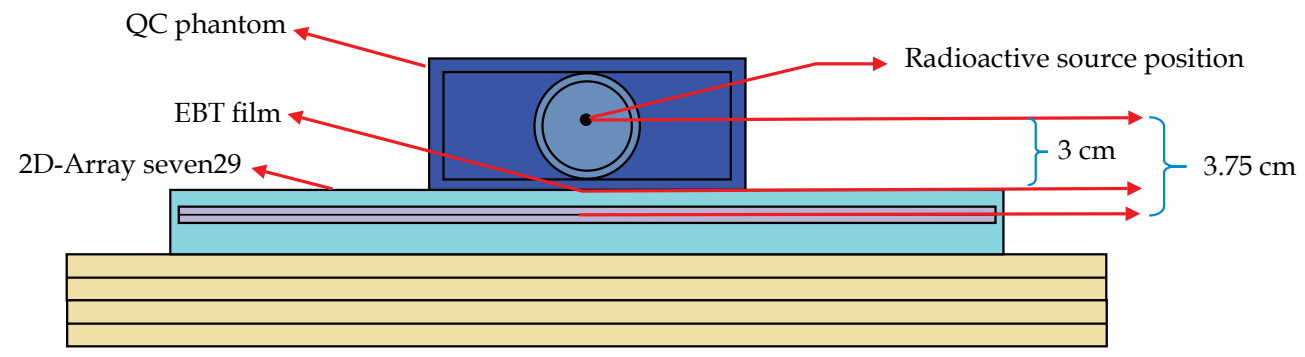

B

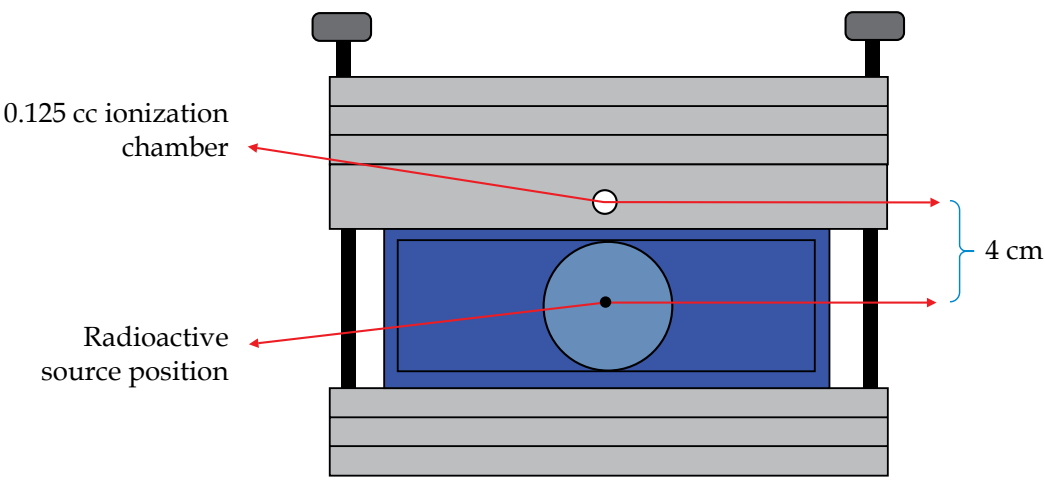

Fig. 4. Measurement setup A) 2D measurement with 2D-Array and EBT3 film and B) point dose measurement with PTW 31010 semiflex ionization chamber 
many) two dimensional (2D) ionization chamber system (Figure 4A). All measurements were repeated 3 times for all step sizes and in every measurement the catheter was removed and re-inserted into the transfer channel to evaluate the reproducibility of the insertion and source position. In the analysis, the gamma evaluation method was used under the criteria of $1 \mathrm{~mm}$ distance to agreement (DTA) and 1\% dose difference (DD) of local dose. As software PTW VeriSoft v.5.1 (PTW-Freiburg, Germany) was used and relative dose distributions were compared during the analysis. In the third step, linearity of the relative dose with changing time was tested in order to evaluate the linearity of the HDR BRT unit for different compensator materials. Linearity test measurements were taken using PTW 31010 semiflex ionization chambers (PTW-Freiburg, Germany) at two different stages. In the first stage the radioactive source was sent to a certain position within the novel IMBRT applicator and irradiations were carried out in 6 different periods of 5, 6, 10, 15, 25 and 35 seconds. In the second stage aluminum, stainless steel and Cerrobend alloy were placed into the modeled QC applicator instead of PLA and similar measurements were repeated for different compensator materials. Distance between the source and ionization chamber was fixed to $4 \mathrm{~cm}$ as shown in Figure 4B. Transit time corrections were applied for all measurements by subtracting 5 seconds from the reading in all measurements and the net reading was obtained. Then, all of the measurements were normalized to 10 seconds net reading to evaluate the linearity of the system for defined conditions. The tolerance value for the linearity was less than $2 \%$.

\section{Dosimetric QC tests}

In the first step of dosimetric QC tests, measurements were performed in homogeneous medium. The IMBRT applicator was placed in the designed QC phantom and the CT of the QC phantom was scanned with $2 \mathrm{~mm}$ slice thickness using a Toshiba Aquilion LB CT (Toshiba Medical Systems, Otowara, Japan) having $100 \mathrm{kVp}$ tube voltage and $350 \mathrm{mAs}$ current value. CT images were then transferred to BrachyVision TPS via a digital imaging and communications in medicine (DICOM) connection. For each designed applicator two different treatment plans including the central CSC and MC in homogeneous medium were created and irradiated in the treatment device. The measurement setup for the 2D dose was set as shown in Figure 4A. 2D dose maps calculated at defined depths were then compared with 2D-Array ionization chamber system and Gafchromic EBT3 film (Ashland Specialty Ingredients, NJ, USA) measurements using gamma analysis method for the local dose. As software PTW VeriSoft v.5.1 was used and relative dose distributions were compared during the analysis. Additionally, point dose measurements were performed with the PTW 31010 semiflex ionization chamber at defined setup conditions as shown in Figure 4B and the measured point dose was compared with the calculated dose in homogeneous medium. In point dose measurement cross-calibration was performed during the use of the PTW 31010 semiflex ionization chamber.
In the second step, the effects of compensator materials on dose attenuation were measured with the QC applicator using the 2D-Array seven29 ionization chamber system. However, the measured dose distribution could not be compared with calculated data in TPS since TG-43 formalism presumes homogeneity of the medium and so the heterogeneity was not taken into account during dose calculation. Therefore compensator materials including aluminum (density: $2.5 \mathrm{~g} / \mathrm{cm}^{3}$ ), stainless steel (density: $8.0 \mathrm{~g} / \mathrm{cm}^{3}$ ) and Cerrobend alloy (density: $9.4 \mathrm{~g} / \mathrm{cm}^{3}$ ) were placed into the anterior region of the applicator and the dose line profile obtained with compensator materials was compared with the PLA-based measurements to evaluate the attenuation properties of the selected materials for a designed compensator geometry.

\section{Results}

\section{Printing accuracy of $3 D$ printer}

The measured differences between the modeled and the printed dimensions of all the products were within $0.5 \mathrm{~mm}( \pm 0.1 \mathrm{~mm}$ resolution of the Vernier caliper). Printed dimensions of the external body and internal body were on average $0.4 \mathrm{~mm}$ (range: $0.3-0.5 \mathrm{~mm}$ ) greater than modeled and dimensions of the transfer channels were on average $0.2 \mathrm{~mm}$ (range: 0-0.3 $\mathrm{mm}$ ) smaller than the designed ones. However, a snug fit of the 5-French catheters in the printed channels was ensured. In addition to printing accuracy, total printing time was evaluated for defined conditions (printing resolution: $0.2 \mathrm{~mm}$, infill percentage: $100 \%$, etc.) and it was almost 20 hours for all parts of the applicator.

\section{Mechanical QC tests}

Source dwell positions measured by EDR2 films were found to be in good agreement with reference $x$-ray marker position and the positional differences were smaller than $0.5 \mathrm{~mm}$. Additionally, the actual catheter insertion position obtained with autoradiography was well matched ( $\leq 1 \mathrm{~mm}$ along the axis) with the reconstructed catheter position in the TPS. The reproducibility of the catheter and source positions for different step sizes was analyzed and 11 of the 12 measurements had a greater than $99 \%$ passing rate for $1 \mathrm{~mm}$ DTA and 1\% DD criteria (Table 1). The lowest gamma passing rate was $97.7 \%$ for $0.1 \mathrm{~mm}$ step size when a comparison was made between 3 consecutive measurements. The linearity values for different applicators and selected compensators were found to be less than $1.5 \%$ (Table 2 ).

\section{Dosimetric QC tests}

Point doses measured in homogeneous medium were found to be compatible with calculated doses in the TPS. The maximum difference was $3.8 \%$, as presented in Table 3 . This difference was first thought to be due to deviation in the catheter and radioactive source position. In fact even $1 \mathrm{~mm}$ deviation from the real position could create dose differences up to $10 \%$ depending on the dose gradient in treatment planning. To minimize this problem a QC phantom was designed and measurements were 
Table 1. Reproducibility of the catheter and source position for different step sizes

\begin{tabular}{lcccc}
\multirow{2}{*}{$\begin{array}{l}\text { Step size } \\
\mathrm{cm})\end{array}$} & \multicolumn{2}{c}{$30 \mathrm{~mm}$ diameter applicator } & \multicolumn{2}{c}{$35 \mathrm{~mm}$ diameter applicator } \\
\cline { 2 - 5 } & Ref. and 2. irradiation & Ref. and 3. irradiation & Ref. and 2. irradiation & Ref. and 3. irradiation \\
\hline 0.1 & $99.10 \%$ & $97.70 \%$ & $100.0 \%$ & $99.70 \%$ \\
\hline 0.5 & $100.0 \%$ & $100.0 \%$ & $100.0 \%$ & $99.20 \%$ \\
\hline 1.0 & $100.0 \%$ & $100.0 \%$ & $100.0 \%$ & $99.70 \%$
\end{tabular}

${ }^{*}$ Gamma analysis criteria: $1 \mathrm{~mm}$ DTA and $1 \%$ DD

Table 2. Linearity value for novel IMBRT applicator and different compensator materials

\begin{tabular}{lccccccc}
$\begin{array}{l}\text { Time } \\
(\mathrm{s})\end{array}$ & Linearity & Linearity & Linearity & Linearity & Linearity & Linearity & Linearity \\
\cline { 2 - 8 } & $\begin{array}{c}30 \mathrm{~mm} \\
\text { applicator }\end{array}$ & $\begin{array}{c}35 \mathrm{~mm} \\
\text { applicator }\end{array}$ & QC applicator & QC applicator & QC applicator & QC applicator & QC applicator \\
\hline 5 & NA & NA & NA & NA & NA & NA & NA \\
\hline 6 & 1.0036 & 1.0118 & 1.0041 & 0.993 & 0.9853 & 0.9885 & 0.9892 \\
\hline 10 & 1.0043 & 0.9881 & 1.0099 & 0.9946 & 1.0064 & 0.9987 & 1.0000 \\
\hline $15^{*}$ & 1.0000 & 1.0000 & 1.0000 & 1.0000 & 1.0000 & 1.0000 & 1.0000 \\
\hline 25 & 1.0007 & 0.9985 & 1.0008 & 1.0015 & 1.0000 & 1.0025 & 0.9928 \\
\hline 35 & 1.0029 & 0.9919 & 1.0058 & 0.993 & 1.0018 & 1.0000 & 0.9964
\end{tabular}

${ }^{*}$ All calculated one second readings were normalized to calculated net reading taken in $15 \mathrm{~s}$

Table 3. Point dose measurements in homogeneous medium

\begin{tabular}{lcccc} 
& \multicolumn{2}{c}{$30 \mathrm{~mm}$ diameter applicator } & \multicolumn{2}{c}{$35 \mathrm{~mm}$ diameter applicator } \\
\cline { 2 - 5 } & CSC & MC & CSC & MC \\
\hline TPS (cGy) & 208.5 & 215.2 & 219.6 & 220.3 \\
\hline Measurements (cGy) & 213.6 & 223.4 & 222.4 & 228.1 \\
\hline Dose differences (\%) & 2.4 & 3.8 & 1.3 & 3.5
\end{tabular}

performed in a fixed geometry. However, there was still positional uncertainty due to the source dwell positioning accuracy, which was lower than $0.5 \mathrm{~mm}$ as measured in the mechanical QC test. The second possibility for this difference was that although the phantom used in the QC tests was produced from water equivalent material, the water equivalent distance (WED) might differ slightly from the physical distance (PD) due to the minimal density differences. PD was arranged as $4 \mathrm{~cm}$ in the QC phantom in the measurements but measured WED was found to be $3.7 \mathrm{~cm}$ in the TPS. Therefore as presented in Table 3 measured point doses were found to be somewhat higher than TPS values for all treatment plans since TG-43 formalism calculated dose according to the PD of $4 \mathrm{~cm}$

In $2 \mathrm{D}$ dose analysis measurements were taken using the 2D-Array ionization chamber system and EBT3 Gafchromic film at $3.75 \mathrm{~cm}$ and $3 \mathrm{~cm}$ distance from the source axis, respectively (Figure 4A). The 2D dose analysis results and the dose distributions obtained at the relevant depth in the TPS were compared with the gamma analysis method. As criteria of gamma analysis four different DTA and DD values $(3 \mathrm{~mm} / 3 \%, 3 \mathrm{~mm} / 5 \%, 5 \mathrm{~mm} / 3 \%$ and $5 \mathrm{~mm} / 5 \%$ ) were compared. The results obtained with both dosimetric systems are presented in Tables 4 and 5. All of the measurement results were above the tolerance value of $90 \%$ gamma passing rate.

Attenuation characteristics of the compensator materials in heterogeneous medium are illustrated in Figures 5-7. In modeled geometry, aluminum, stainless steel and Cerrobend alloy attenuated the dose more than $15 \%, 35 \%$ and $75 \%$ with respect to PLA respectively. Attenuation ratios of the materials were found to be associated with density of the compensator as expected.

\section{Discussion}

BRT significantly increases loco-regional control when applied alone or combined with EBRT in gynecological malignancies [6,7]. The main beneficial effect of BRT is that it provides better organ sparing of the surrounding tissues due to the rapid dose fall off with distance from the target volume $[8,9]$.

Vaginal BRT alone or combined with EBRT in EC is being widely used in the adjuvant setting and the techni- 
Table 4. Comparison of TPS and 2D-Array measurement in homogeneous medium

\begin{tabular}{lcccc} 
Analysis criteria & \multicolumn{2}{c}{$30 \mathrm{~mm}$ diameter applicator } & 35 mm diameter applicator \\
\cline { 2 - 5 } (DTA and DD) & CSC & MC & CSC & MC \\
\hline $3 \mathrm{~mm}$ and 3\% & $94.8 \%$ & $98.5 \%$ & $95.0 \%$ & $100.0 \%$ \\
\hline $3 \mathrm{~mm}$ and 5\% & $97.4 \%$ & $95.8 \%$ & $100.0 \%$ & $98.2 \%$ \\
\hline $5 \mathrm{~mm}$ and 3\% & $100.0 \%$ & $99.2 \%$ & $100.0 \%$ & $100.0 \%$ \\
\hline $5 \mathrm{~mm}$ and 5\% & $100.0 \%$ & & & $100.0 \%$
\end{tabular}

Table 5. Comparison of TPS and EBT3 film measurement in homogeneous medium

\begin{tabular}{|c|c|c|c|c|}
\hline \multirow{2}{*}{$\frac{\text { Analysis criteria }}{\text { (DTA and DD) }}$} & \multicolumn{2}{|c|}{$30 \mathrm{~mm}$ diameter applicator } & \multicolumn{2}{|c|}{$35 \mathrm{~mm}$ diameter applicator } \\
\hline & $\mathrm{CSC}$ & MC & $\mathrm{CSC}$ & MC \\
\hline $3 \mathrm{~mm}$ and $3 \%$ & $93.0 \%$ & $99.3 \%$ & $92.7 \%$ & $93.7 \%$ \\
\hline $3 \mathrm{~mm}$ and $5 \%$ & $94.3 \%$ & $99.7 \%$ & $95.8 \%$ & $97.1 \%$ \\
\hline $5 \mathrm{~mm}$ and $3 \%$ & $94.4 \%$ & $99.9 \%$ & $96.3 \%$ & $95.8 \%$ \\
\hline $5 \mathrm{~mm}$ and $5 \%$ & $94.6 \%$ & $99.9 \%$ & $97.7 \%$ & $97.6 \%$ \\
\hline
\end{tabular}

cal developments in the computer era led to replacement of $2 \mathrm{D}$ radiography-based treatment planning by CTbased treatment modalities, with improvements in imag-

$\mathbf{A}$
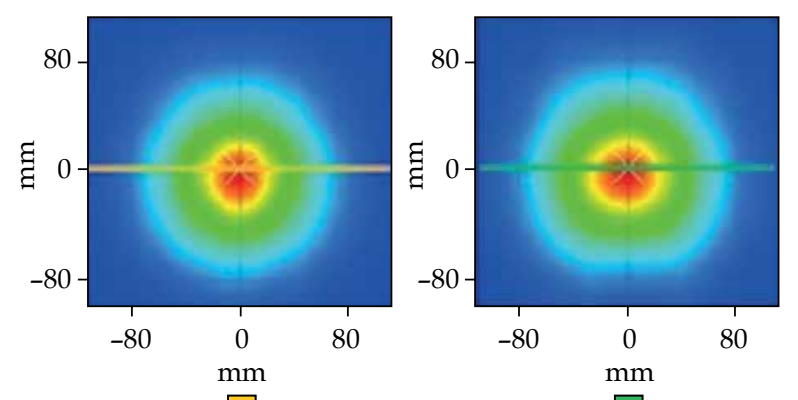

C

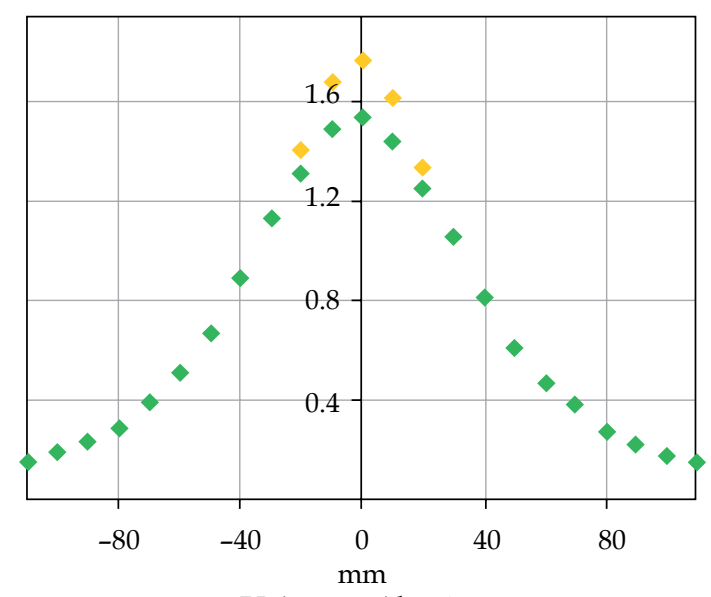

$\square$ PLA $\square$ Aluminum ing quality and availability. In conventional techniques it was mostly recommended that dose prescription should be made at a depth of $5 \mathrm{~mm}$ from the applicator surface
B

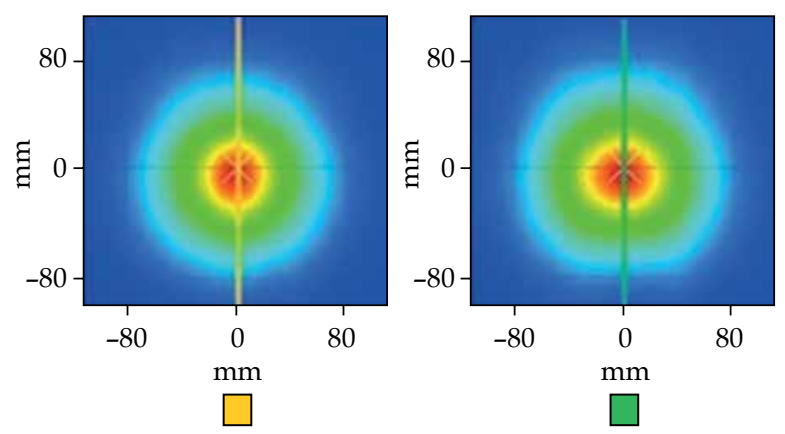

D

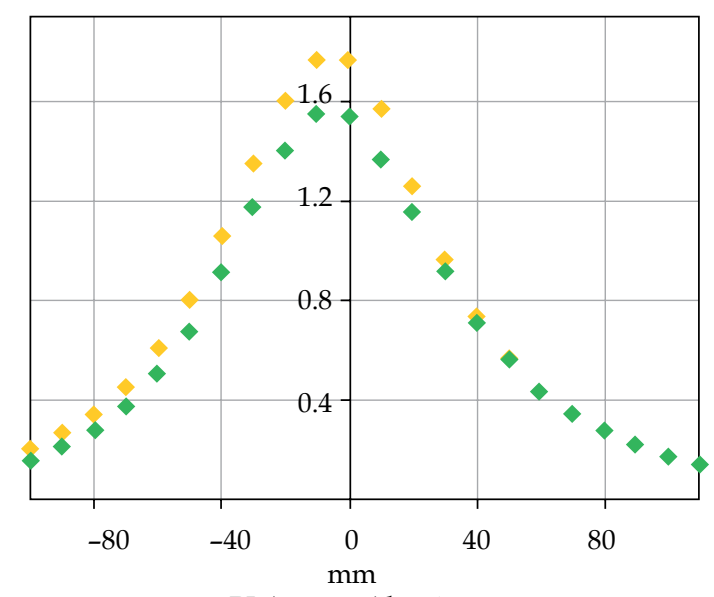

$\square$ PLA $\square$ Aluminum

Fig. 5. Measured dose map A) in x axis, B) in y axis and line dose graphic C) in x axis, D) in y axis for PLA (yellow) and aluminum (green) 
A
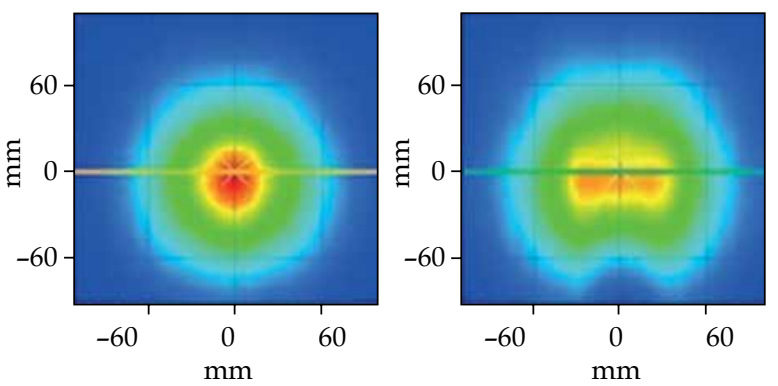

C

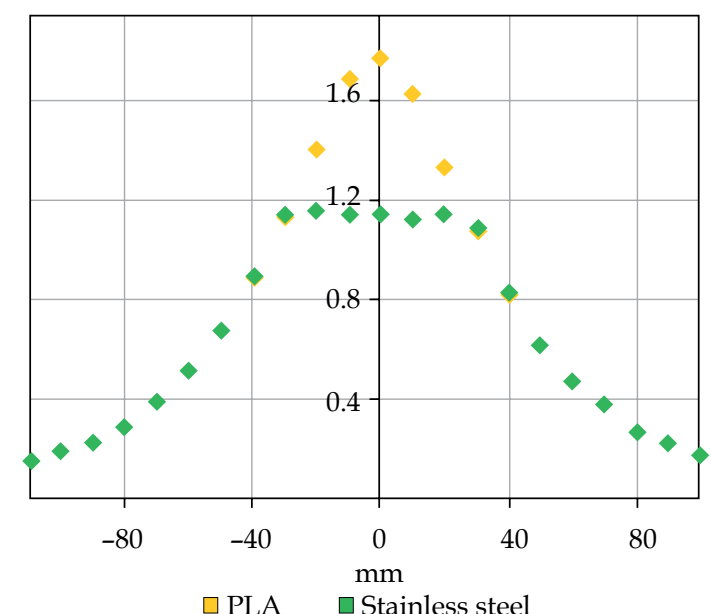

B
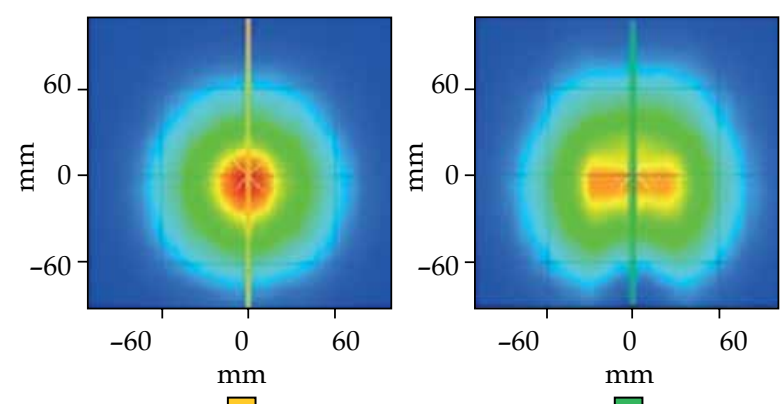

D

Gy

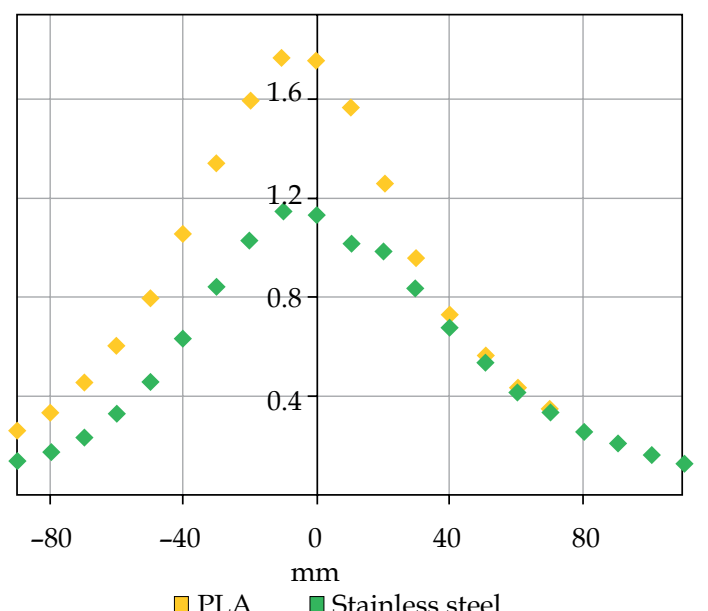

Fig. 6. Measured dose map A) in x axis, B) in y axis and line dose graphic C) in x axis, D) in y axis for PLA (yellow) and stainless steel (green)

or to the surface [2]. Uncertainties due to patient anatomy and tumor size have been neglected, which may lead to overdoses or underdoses in the target and toxic doses to the critical organs in the vicinity. With the advent of three dimensional brachytherapy (3DBRT), several studies have been carried out to eliminate the uncertainties in gynecological BRT. In a study performed by Kim et al. [16] it was reported that the use of 3DBRT modalities in vaginal cuff irradiation could significantly reduce the rectum and bladder doses without sacrificing the target dose. In a similar study by Onal et al. [17], it was found that $43 \%$ of the patients treated with a cylinder applicator had air gaps between the applicator surface and vaginal mucosa that caused dose uncertainty. It was also reported that $6.3 \%$ of these patients did not take the prescribed dose when the dose prescription was made to the depth of $5 \mathrm{~mm}$ from the applicator surface.

In the last two decades the developments in imaging, pathology, surgery, radiotherapy and systemic treatment have given rise to early diagnosis and better delineation of the disease, which led to effective treatment with high cure rates in patients with gynecological cancer. It is becoming increasingly important to reduce the treatment-related side effects in these patients with long survival expectancy. Rectum, bladder and sigmoid are the major dose-limiting organs in the treatment of gyneco- logic malignancies $[2,12,15,18]$, particularly in patients with locally advanced disease when there is a need for combining intracavitary BRT with EBRT due to the large tumor volume. In order to overcome the problems in gynecological BRT, MC applicators with different designs and trademarks, such as Capri, which is a trademark of Varian Medical Systems, Inc. have been introduced by the manufacturers. Iftimia et al. [12], Demanes et al. [13] and Tanderup et al. [14] reported that MC applicators had dosimetric advantages in terms of target coverage and critical organ doses compared with CSC applicators. Additionally, Iftimia et al. [12], Mendez et al. [19] and Shin et al. [20] reported that MC applicators may be used instead of interstitial BRT in small lesions up to $1 \mathrm{~cm}$ in depth. However, in the case of increased tumor size and depth, existing MC applicators lose their effectiveness. Although interstitial BRT has a great advantage in selected patients, the necessity of operating room conditions during application and a postoperative care unit after treatment are the major disadvantages. In this study we proposed a novel compensator-based IMBRT applicator system to eliminate these disadvantages in selected patient groups. One of the most important advantages of this applicator is that the special design of the applicator allows different combinations of the source transfer channels and desired lengths of compensator materials during application and treatment. 
A
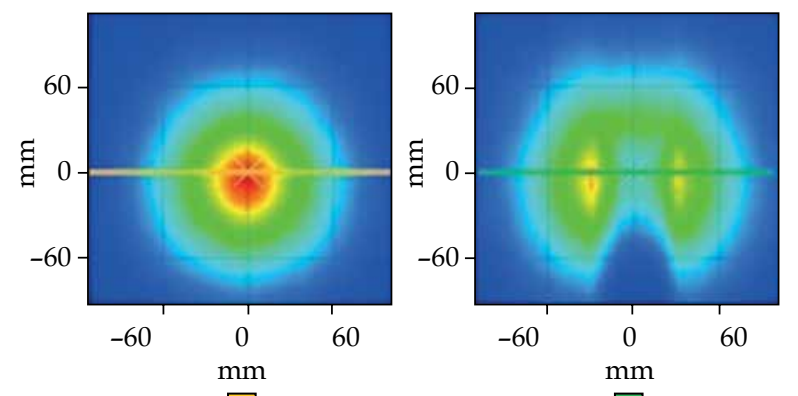

C

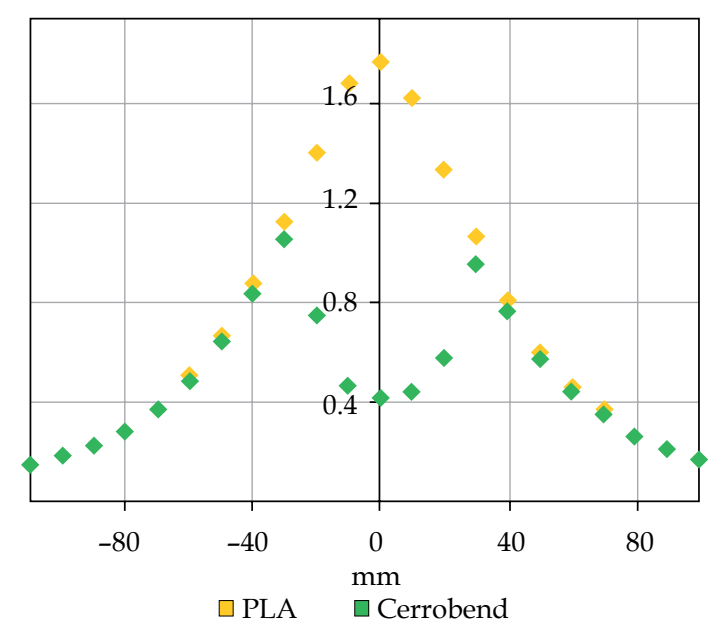

B
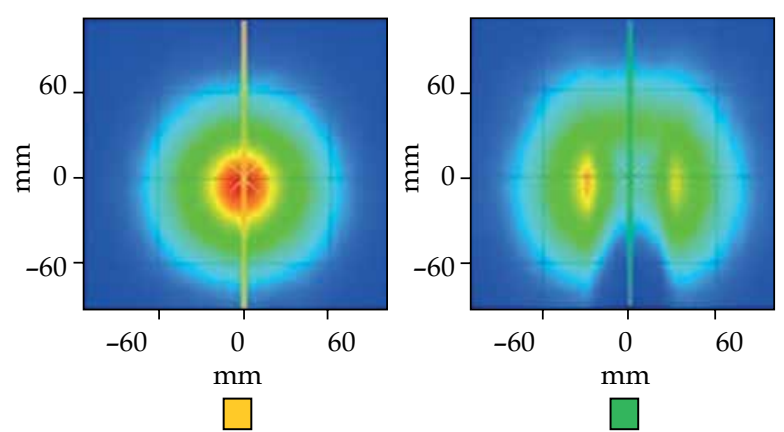

D

Gy

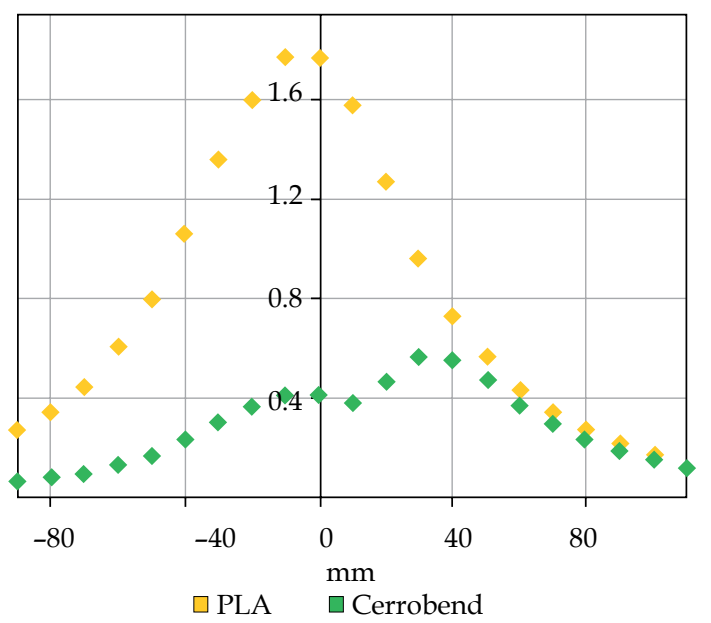

Fig. 7. Measured dose map A) in x axis, B) in y axis and line dose graphic C) in $x$ axis, D) in y axis for PLA (yellow) and Cerrobend (green)

Additionally, this novel applicator system will allow a simultaneous integrated boost dose to the tumor or irradiation of more than two different treatment plans consecutively in the same treatment fraction without changing the position of the external part of the applicator. With this aspect treatment plans created with the IMBRT applicator can be an alternative to interstitial BRT even at a depth of more than $1 \mathrm{~cm}$. In addition to all these advantages the invasive procedure required by the interstitial BRT can be eliminated in a selected group of patients and treatment comfort can be increased without compromising the quality of the treatment planning.

3D printers have been widely used in the production of compensator materials for proton therapy [21,22], bolus [23,24,25,26,27], QC phantom for EBRT [28,29,30,31,32], applicators and auxiliary equipment for BRT facilities [33,34,35,36,37,38,39,40]. Ju et al. [21] and Zou et al. [22] stated that the compensators produced with PLA material had similar dosimetric and physical properties with conventional compensators used in proton therapy. In EBRT facilities on the other hand, Park et al. [22] showed that a patient-specific bolus could be produced using 3D printer technology in patients with an irregular body contour. In that way it was emphasized that daily setup uncertainty and dose differences due to the bolus placement could be minimized using a 3D printer-based patient-specific bolus.
Ehler et al. [32] reported that patient-specific QC phantoms could also be printed for RT facilities. In addition, similar to our study, Harris et al. [33] and Jones et al. [34] showed that a low-cost and patient-specific mould BRT applicator could be manufactured using a 3D printer. In another study Sethi et al. [36] evaluated treatment plans of three different patients and emphasized that the limited size of commercially available applicators could be eliminated by using 3D printing technology and effectiveness of the treatment plans could be increased by producing a patient-specific applicator. Additionally, Cunha et al. [35] and Sethi et al. [36] produced a cylindrical applicator for vaginal cuff applications using 3D printer-based technology.

In $3 \mathrm{D}$ printers it is possible to use different filament materials such as ABS, PLA, polycarbonate-ISO (PC-ISO), nylon, PET, carbon fiber, aluminum and wood according to the printer model and the area of use. For medical purposes printing material can be selected depending on several characteristics of the filament including sterilization, biocompatibility, non-toxicity, flexibility and durability. In the production of the BRT applicator, materials should be tissue equivalent for TG-43 formalism, sterilized, biocompatible, non-toxic and with strength for clinical use. Cunha et al. [35] used PC-ISO filament with a density of $1.2 \mathrm{~g} / \mathrm{cm}^{3}$ in the production of the applicator and showed that the measurements were compatible with TPS. Atha- 
nasiou et al. [41] and Rankin et al. [42] stated that PLA material is suitable for medical applications and ethylene oxide (EtO) and glutaraldehyde solution can be used for sterilization. In our study PLA was used as a filament material with a density of $1.25 \mathrm{~g} / \mathrm{cm}^{3}$ and we also found that measurements taken in homogeneous medium were consistent with TPS for PLA material.

There are several limitations of our study. The first one is that the calculation algorithm used in the TPS was based on TG-43 formalism. We could not take into account the heterogeneity and could not analyze the effects of compensator materials on treatment planning. The second limitation is that this study was only focused on mechanical and dosimetric feasibility of the novel IMBRT applicator without any chance to test it in patients. In the next study two sets of measurements will be performed to eliminate these limitations. In the first step simulation plans with heterogeneity correction will be created in a QC phantom and verification of the treatment planning will be performed using appropriate dosimetry systems such as EBT3 Gafchromic film. In the second step simulation plans will be created based on real clinical cases and a prospective clinical study will be designed to analyze the effectiveness of the system in suitable patient groups.

\section{Conclusions}

This novel IMBRT applicator has met the necessary requirements in mechanical and dosimetric $\mathrm{QC}$ tests in homogeneous medium. In the next stage simulation plans with heterogeneity correction will be created in a QC phantom and a prospective trial will be planned in appropriate patient groups so that the clinical effectiveness of the applicator can be evaluated.

\section{Acknowledgements}

Fatih Biltekin and H. Fadil Akyol equally contributed to this article. Hacettepe Technology Transfer Center (HT-TTM) Patent Office provided expert support for intellectual property rights (IPR) and financial support for patent application. The ownership right of the applicator belongs to Hacettepe University and H. Fadil Akyol, MD and Fatih Biltekin, PhD are inventors of the novel system. This invention entitled the "Vaginal Intensity Modulated Brachytherapy (IMBT) Applicator" was awarded a silver medal at TEKNOFEST 2019 Istanbul- ISIF'19, $4^{\text {th }}$ Istanbul International Invention Fair.

\section{Disclosure}

The authors report no conflict of interest.

\section{References}

1. Banerjee R, Kamrava M. Brachytherapy in the treatment of cervical cancer: a review. Int J Womens Health 2014; 6: 555-564.

2. Sabater S, Andres I, Lopez-Honrubia V et al. Vaginal cuff brachytherapy in endometrial cancer - a technically easy treatment? Cancer Manag Res 2017; 9: 351-362.

3. Zakem SJ, Robin TP, Smith DE et al. Evolving trends in the management of high-intermediate risk endometrial cancer in the United States. Gynecol Oncol 2019; 152: 522-527.
4. Holschneider $\mathrm{CH}$, Petereit DG, Chu C et al. Brachytherapy: A critical component of primary radiation therapy for cervical cancer: For the Society of Gynecologic Oncology (SGO) and the American Brachytherapy Society (ABS). Brachytherapy 2019; 18: 123-132.

5. Gill BS, Lin JF, Krivak TC et al. National Cancer Data Base analysis of radiation therapy consolidation modality for cervical cancer: the impact of new technological advancements. Int J Radiat Oncol Biol Phys 2014; 90: 1083-1090.

6. Horowitz NS, Peters WA, Smith MR et al. Adjuvant high dose rate vaginal brachytherapy as treatment of stage I and II endometrial carcinoma. Obstet Gynecol 2002; 99: 235-240.

7. Pearcey RG, Petereit DG. Post-operative high dose rate brachytherapy in patients with low to intermediate risk endometrial cancer. Radiother Oncol 2000; 56: 17-22.

8. Zhang H, Donnelly ED, Strauss JB et al. Therapeutic analysis of high-dose-rate 192Ir vaginal cuff brachytherapy for endometrial cancer using a cylindrical target volume model and varied cancer cell distributions. Med Phys 2016; 43: 483-494.

9. Shwetha B, Ravikumar M, Palled SR et al. Dosimetric comparison of high dose rate brachytherapy and intensity-modulated radiation therapy for cervical carcinoma. J Med Phys 2011; 36: 111-116.

10. Kemikler G. Post-op brakiterapide fizik özellikler. Turk J Oncol 2012; 27 (Suppl 1): 50-54.

11. Wortman BG, Creutzberg CL, Putter H et al. Ten-year results of the PORTEC-2 trial for high-intermediate risk endometrial carcinoma: improving patient selection for adjuvant therapy. Br J Cancer 2018; 119: 1067-1074.

12. Iftimia I, Cirino ET, Mower HW et al. Treatment planning methodology for the Miami Multichannel Applicator following the American Brachytherapy Society recently published guidelines: the Lahey Clinic experience. J Appl Clin Med Phys 2013; 14: 214-227.

13. Demanes DJ, Rege S, Rodriques RR et al. The use and advantages of a multichannel vaginal cylinder in high-dose-rate brachytherapy. Int J Radiat Oncol Biol Phys 1999; 44: 211-219.

14. Tanderup K, Lindegaard JC. Multi-channel intracavitary vaginal brachytherapy using three-dimensional optimization of source geometry. Radiother Oncol 2004; 70: 81-85.

15. Beriwal S, Bhatnagar A, Heron DE et al. High-dose-rate interstitial brachytherapy for gynecologic malignancies. Brachytherapy 2006; 5: 218-222.

16. Kim $\mathrm{H}$, Kim $\mathrm{H}$, Houser $\mathrm{C}$ et al. Is there any advantage to three-dimensional planning for vaginal cuff brachytherapy? Brachytherapy 2012; 11: 398-401.

17. Onal C, Guler OC, Dolek Y. The impact of air pockets around the vaginal cylinder on vaginal vault brachytherapy. $\mathrm{Br} J \mathrm{Ra}$ diol 2015; 88: 20140694.

18. Harkenrider MM, Block AM, Alektiar KM et al. American Brachytherapy Task Group Report: Adjuvant vaginal brachytherapy for early-stage endometrial cancer: A comprehensive review. Brachytherapy 2017; 16: 95-108.

19. Mendez LC, Paudel M, Wronski M et al. Dosimetric comparison of interstitial brachytherapy with multi-channel vaginal cylinder plans in patients with vaginal tumors. Radiat Oncol 2017; 12: 84.

20. Shin SM, Duckworth TL, Cooper BT et al. Use of a flexible inflatable multi-channel applicator for vaginal brachytherapy in the management of gynecologic cancer. Front Oncol 2015; 5: 201.

21. Ju SG, Kim MK, Hong CS et al. New technique for developing a proton range compensator with use of a 3-dimensional printer. Int J Radiat Oncol Biol Phys 2014; 88: 453-458.

22. Zou W, Fisher T, Zhang M et al. Potential of 3D printing technologies for fabrication of electron bolus and proton compensators. J Appl Clin Med Phys 2015; 16: 4959. 
23. Su S, Moran K, Robar JL. Design and production of 3D printed bolus for electron radiation therapy. J Appl Clin Med Phys 2014; 15: 4831.

24. Kim SW, Shin HJ, Kay CS et al. A customized bolus produced using a 3-dimensional printer for radiotherapy. PLoS One 2014; 9: e110746.

25. Burleson S, Baker J, Hsia AT et al. Use of 3D printers to create a patient-specific 3D bolus for external beam therapy. J Appl Clin Med Phys 2015; 16: 166-178.

26. Park SY, Choi CH, Park JM et al. A patient-specific polylactic acid bolus made by a 3D printer for breast cancer radiation therapy. PLoS One 2016; 11: e0168063.

27. Park JW, Yea JW. Three-dimensional customized bolus for intensity modulated radiotherapy in a patient with Kimura's disease involving the auricle. Cancer Radiother 2016; 20: 205-209.

28. Gear JI, Long C, Rushforth D et al. Development of patient-specific molecular imaging phantoms using a 3D printer. Med Phys 2014; 41: 082502

29. Kairn T, Crowe SB, Markwell T. Use of 3D printed materials as tissue equivalent phantoms. In: World Congress on Medical Physics and Biomedical Engineering, June 7-12, 2015 Toronto, Canada. Springer International Publishing; 2015; 728-731.

30. Bieniosek MF, Lee BJ, Levin CS. Technical note: characterization of custom 3D printed multimodality imaging phantoms. Med Phys 2015; 42: 5913-5918.

31. Madamesila J, McGeachy P, Barajas JEV et al. Characterizing $3 \mathrm{D}$ printing in the fabrication of variable density phantoms for quality assurance of radiotherapy. Phys Med 2016; 32: 242-247.

32. Ehler ED, Barney BM, Higgins PD et al. Patient specific 3D printed phantom for IMRT quality assurance. Phys Med 2014; 59: 5763.

33. Harris BD, Nilsson S, Poole CM. A feasibility study for using ABS plastic and a low-cost 3D printer for patient-specific brachytherapy mould design. Australas Phys Eng Sci Med 2015; 38: 399-412.

34. Jones EL, Baldion AT, Thomas C et al. Introduction of novel 3D-printed superficial applicators for high-dose-rate skin brachytherapy. Brachytherapy 2017; 16: 409-414.

35. Cunha JAM, Mellis K, Sethi R et al. Evaluation of PC-ISO for customised, 3D printed, gynecologic 192-Ir HDR brachytherapy applicators. J Appl Clin Med Phys 2015; 16: 5168.

36. Sethi R, Cunha A, Mellis K et al. Clinical applications of custom-made vaginal cylinders constructed using three dimensional printing technology. J Contemp Brachytherapy 2016; 8: 208-214.

37. Lindegaard JC, Madsen ML, Traberg A et al. Individualised 3D printed vaginal template for MRI guided brachytherapy in locally advanced cervical cancer. Radiother Oncol 2016; 118: 173-175.

38. Poulin E, Gardi L, Fenster A et al. Towards real-time 3D ultrasound planning and personalized 3D printing for breast HDR brachytherapy treatment. Radiother Oncol 2015; 114: 335-338.

39. Walker JM, Elliott DA, Kubicky CD et al. Manufacture and evaluation of 3D printed sizing tools for use during intraoperative breast brachytherapy. Adv Radiat Oncol 2016; 1: 132-135.

40. Huang MW, Zhang JG, Zheng L et al. Accuracy evaluation of a 3D-printed individual template for needle guidance in head and neck brachytherapy. J Radiat Res 2016; 57: 662-667.

41. Athanasiou KA, Niederauer GG, Agrawal CM. Sterilization, toxicity, biocompatibility and clinical applications of polylactic acid/polyglycolic acid copolymers. Biomaterials 1996; 17: 93-102.
42. Rankin TM, Giovinco NA, Cucher DJ et al. Three-dimensional printing surgical instruments: are we there yet? J Surg Res 2014; 189: 193-197. 